\title{
Capn4 regulates migration and invasion of ovarian carcinoma cells via targeting osteopontin-mediated PI3K/AKT signaling pathway
}

\author{
YUANYUAN CHEN, GANG WANG, YINGMEI WANG, XIAOLI GAO, KAN WANG, JIE LI and FENGXIA XUE \\ Department of Gynecology and Obstetrics, Tianjin Medical University General Hospital, Tianjin 300052, P.R. China
}

Received October 21, 2017; Accepted August 15, 2018

DOI: $10.3892 / \mathrm{ol} .2018 .9524$

\begin{abstract}
Previous studies have demonstrated that calpain small subunit 4 (Capn4) is able to regulate the viability and metastasis of cancer cells. However, the regulatory effects and underlying molecular mechanism of Capn4 in ovarian carcinoma cells are not well understood. The purpose of the present study was to investigate the role of Capn4 in ovarian carcinoma cells and analyze the possible mechanism mediated by Capn 4 . The expression levels of Capn4 and osteopontin (OPN) were determined and the phosphoinositide 3-kinase (PI3K)/protein kinase B (AKT) signaling pathway was analyzed in ovarian carcinoma cells. The results of the present study revealed that Capn4 and OPN were overexpressed in clinical ovarian carcinoma tissues and ovarian carcinoma cells. Capn4 silencing downregulated OPN expression, and suppressed ovarian carcinoma cell viability and migration. Capn 4 silencing enhanced apoptosis of ovarian carcinoma cells by increasing activity of the capase- 3 apoptosis signaling pathway. Capn 4 promoted the metastasis of ovarian carcinoma cells by interacting with the PI3K/AKT signaling pathway via upregulation of OPN expression. In conclusion, the results of the present study indicate that Capn 4 may be a potential therapeutic target for the treatment of ovarian carcinoma.
\end{abstract}

\section{Introduction}

Ovarian cancer is the most common gynecological malignancy, with the highest mortality among all gynecological malignancies (1). A previous study demonstrated that the incidence of ovarian cancer is increasing globally (2). Epithelial ovarian cancer is a distinct histotype of ovarian cancer that is characterized by poor response rates to chemotherapies in

Correspondence to: Professor Fengxia Xue, Department of Gynecology and Obstetrics, Tianjin Medical University General Hospital, 154 Anshan Road, Tianjin 300052, P.R. China

E-mail: medicine1956@163.com

Key words: calpain small subunit 4, osteopontin, ovarian carcinoma, migration, invasion, phosphoinositide 3-kinase/protein kinase B the advanced stages of disease (3). Using a meta-analysis of prevalence rates, it was identified that females with ovarian cancer who had undergone chemotherapy treatment demonstrated continued symptoms of depression and anxiety (4). Ineffective or prolonged management of treatments may contribute to the worsening of symptoms, treatment noncompliance and even a decrease in health-associated quality of life (5). Although previous studies have proposed certain improvements in early diagnosis and anticancer therapies for ovarian carcinoma (6-9), metastasis remains the major challenge in the treatment of ovarian carcinoma. Therefore, it is imperative to understand the mechanisms of the metastasis in this disease.

Novel strategies for ovarian carcinoma therapy have attracted attention from clinicians and doctors (10-12). Targeted therapy has exhibited efficient antitumor effects worldwide, and serves a critical role in modern treatment modalities (13-15). Gu et al (16) demonstrated that calpain small subunit 4 (Capn4) may promote progression of non-small cell lung cancer via the upregulation of matrix metalloproteinase 2 expression. Capn 4 overexpression has been observed in and is associated with the invasiveness of hepatocellular carcinoma (17). Notably, Capn4 may promote epithelial ovarian carcinoma metastasis through osteopontin (OPN)-mediated activation of the $\mathrm{Wnt} / \beta$-catenin pathway (18). Study also found that caspase-3 up-regulation strongly suppressed tumor growth and improved mouse survival with little liver toxicity (19). However, the associations between Capn 4 and the caspase- 3 signaling pathway have not been completely elucidated.

OPN is an integrin-binding matrix phosphorylated glycoprotein, which is frequently overexpressed in a number of advanced human cancer tissues (20). A previous study has indicated that OPN promotes ovarian cancer progression and cell survival through the phosphoinositide 3-kinase $(\mathrm{PI} 3 \mathrm{~K}) /$ protein kinase B (AKT) signaling pathway (21). In addition, OPN expression is a biomarker in ovarian cancer progression, which contributes to the understanding of the physiopathology of ovarian cancer progression and tumorigenesis (22). Furthermore, OPN overexpression has been observed in endometrioid endometrial cancer and ovarian endometrioid cancer (23).

In the present study, the expression levels of Capn4 and OPN in clinical ovarian carcinoma tissues and a cell line were evaluated. It was identified that Capn4 was highly expressed 
in ovarian carcinoma tissues and cell lines compared with normal ovarian tissues and cells. It was also revealed that Capn4 may promote ovarian carcinoma metastasis through the OPN-mediated PI3K/AKT signaling pathway. Taken together, the results of the present study indicated that Capn 4 may be regarded as a novel potential target for ovarian carcinoma therapy.

\section{Materials and methods}

Cell culture and reagents. SKOV3 and normal ovarian epithelial cells (IOSE80) cells were purchased from the Cell Bank of Type Culture Collection of Chinese Academy of Science (Shanghai, China). Cells were cultured in Dulbecco's modified Eagle's medium (DMEM; Gibco; Thermo Fisher Scientific, Inc., Waltham, MA, USA) supplemented with $10 \%$ fetal bovine serum (Gibco; Thermo Fisher Scientific, Inc.) in a $37^{\circ} \mathrm{C}$ humidified atmosphere with $5 \% \mathrm{CO}_{2}$.

Patients and tissue samples. Normal ovarian tissues and ovarian tumor tissues were obtained from 5 patients (42 years; range, 32-68 years) using a biopsy at Tianjin Medical University General Hospital (Tianjin, China) between May 2014 and June 2017. None of the patients received chemotherapy or other antitumor treatment prior to surgery. All patients provided written informed consent. Ethical approval for the use of human tissue samples was provided by the Institutional Research Ethics Committee of Tianjin Medical University General Hospital.

Reverse transcription-quantitative polymerase chain reaction (RT-qPCR) assays. Total RNA from SKOV3 cells, normal ovarian tissues or ovarian tumor tissues was extracted using an RNeasy Mini kit (Qiagen Sciences, Inc., Gaithersburg, MD, USA). The mRNA expression levels of Capn 4 and $\beta$-actin in SKOV3 cells were determined by RT-qPCR (24). All primers were synthesized by Thermo Fisher Scientific, Inc. (Capn4, forward, 5'-ACCCACTCCGTAACCTC-3' and reverse, 5'-GGG TAGCAACCGTGAA-3'; $\beta$-actin forward, 5'-CATCTCTTG CTCGAAGTCCA-3' and reverse, 5'-ATCATGTTTGAGACC TTCAACA-3'). PCR was performed as follows: Preliminary denaturation at $94^{\circ} \mathrm{C}$ for $2 \mathrm{~min}$, followed by 45 cycles of $95^{\circ} \mathrm{C}$ for $30 \mathrm{sec}$, the annealing temperature decreased to $55^{\circ} \mathrm{C}$ for $30 \mathrm{sec}$, and $72^{\circ} \mathrm{C}$ for $10 \mathrm{~min}$ in a total reaction volume of $20 \mu \mathrm{l}$ containing $50 \mathrm{ng}$ genomic DNA, $200 \mu \mathrm{M}$ dNTP, 2.5 units Taq DNA polymerase (Takara Biotechnology Co., Ltd., Dalian, China) and $200 \mu \mathrm{M}$ primers. Relative mRNA expression level changes were calculated using the $2^{-\Delta \Delta \mathrm{Cq}}$ method (25). The results are expressed as the $n$-fold comparison with the $\beta$-actin control.

Lactate dehydrogenase ( $L D H$ ) assay. The ovarian tumor cells SKOV3 were cultured until a $90 \%$ monolayer was formed, and then the medium was removed. The ovarian tumor cells were washed three times and subsequently incubated with Triton X-100 (1\%) for $30 \mathrm{~min}$. LDH activity in the lysates was measured using the Promega CytoTox 96 assay kit (Promega $^{\mathrm{TM}}$ G1780), according to the manufacturer's protocol.

Gene silencing with small interfering RNA (siRNA). SKOV3 cells $\left(4 \times 10^{5}\right.$ cells/well) were seeded in 6 -well plates for $24 \mathrm{~h}$ at $37^{\circ} \mathrm{C}$. The medium was removed and Opti-MEM reduced serum medium (Invitrogen; Thermo Fisher Scientific, Inc.) was added for $24 \mathrm{~h}$ at $37^{\circ} \mathrm{C}$. siRNA-Capn4 (siR-Capn4), siRNA-OPN (siR-OPN) or control scrambled siRNA (siR-vector) were chemically synthesized by Shanghai GenePharma Co., Ltd. (Shanghai, China). The sequences were as follows: siR-Capn4, 5'-GCACCAUGCAGAAUACAAA-3', siRNA-OPN, 5'-CCU GUGCCAUACCAGUUAA-3', siR-vector, 5'-CUCGUCUCA UUGATGACAGTT-3'. SKOV3 cells were transfected with 100 pmol siRNA using Lipofectamine ${ }^{\circledR} 2000$ (Invitrogen; Thermo Fisher Scientific, Inc.), according to the manufacturer's protocol.

Cell invasion and migration assays. SKOV3 cells and Capn4-slienced SKOV3 cells were treated with PI3K inhibitor (SW30-Calbiochem, $200 \mu \mathrm{M}$; Sigma-Aldrich; Merck KGaA, Darmstadt, Germany) for $24 \mathrm{~h}$ at $37^{\circ} \mathrm{C}$. For the invasion assay, SKOV 3 cells were suspended at a density of $1 \times 10^{5}$ in $500 \mu 1$ serum-free DMEM. The cells were then applied to the tops of BD BioCoat Matrigel Invasion Chambers (BD Biosciences, Franklin Lakes, NJ, USA), according to the manufacturer's protocols. For the migration assay, a control insert (BD Biosciences) was used instead of a Matrigel Invasion Chamber. The tumor cells that had invaded or migrated were fixed with $4 \%$ formaldehyde at room temperature for $5 \mathrm{~min}$ and stained with $0.5 \%$ crystal violet (Beijing Solarbio Science \& Technology Co., Ltd., Beijing, China) at room temperature for $15 \mathrm{~min}$. and counted in at least three randomly selected fields of view for each membrane.

Flow cytometry. Apoptosis of the SKOV3 cells was evaluated using an Annexin V-Fluorescein Isothiocyanate (FITC) and Propidium Iodide (PI) Apoptosis Detection kit (BD Biosciences). SKOV3 cells were collected and suspended with annexin V-FITC and PI, according to the manufacturer's protocol. Fluorescence was determined with a fluorescence-activated cell sorting flow cytometer using FCS Express $^{\text {TM }}$ IVD software (version 4; De Novo Software, Los Angeles, CA, USA).

Western blotting. SKOV3 cells $\left(1 \times 10^{6}\right)$ were homogenized in lysate buffer containing protease inhibitor (cat. no. P3480; Sigma-Aldrich; Merck $\mathrm{KGaA}$ ) and were centrifuged at $8,000 \times \mathrm{g}$ at $4^{\circ} \mathrm{C}$ for $10 \mathrm{~min}$. The supernatant was used for analyzing protein expression. Protein concentration was measured using a bicinchoninic acid protein assay kit (Thermo Fisher Scientific, Inc.). Protein samples $(20 \mu \mathrm{g})$ were separated by SDS-PAGE (12\% gel) and transferred onto polyvinylidene difluoride membranes (EMD Millipore, Billerica, MA, USA), and incubated with rabbit anti-mouse primary antibodies against PI3K (1:1,000; cat. no. ab151549), phospho-(p-)PI3K (1:1,000; cat. no. ab86714), AKT (1:1,000; cat. no. ab8805), p-AKT (1:1,000; cat. no. ab133458), Capn4 (1:1,000; cat. no.ab28237), OPN (1:1,000; cat.no.ab8448), caspase-3 (1:1,000; cat. no. ab13847), caspase-9 (1:1,000; cat. no. ab52298), B-cell lymphoma (Bcl)-2 (1:1,000; cat. no. ab692), Bcl-w (1:1,000; cat. no. ab32370) and $\beta$-actin (1:1,000; cat. no. ab5262) (all Abcam, Cambridge, UK). Horseradish peroxidase (HRP)-conjugated goat anti-rabbit immunoglobulin G (cat. no. PV-6001; OriGene Technologies, Inc., Rockville, MD, USA) was added for $24 \mathrm{~h}$ 
A

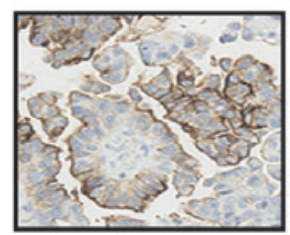

Normal ovarian epithelial tissues

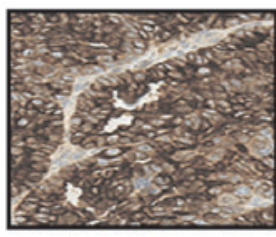

Ovarian carcinoma
B

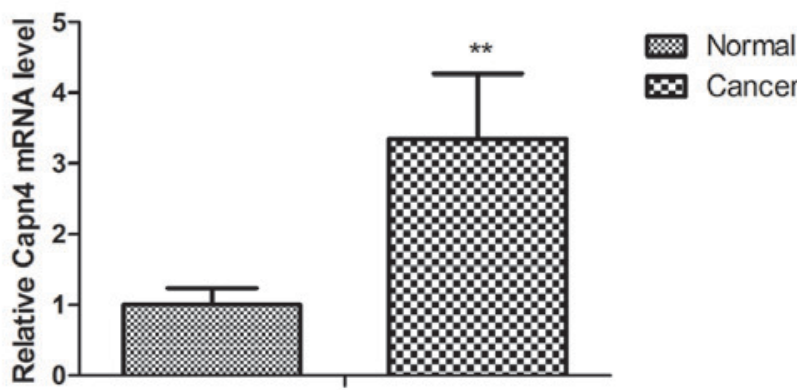

C
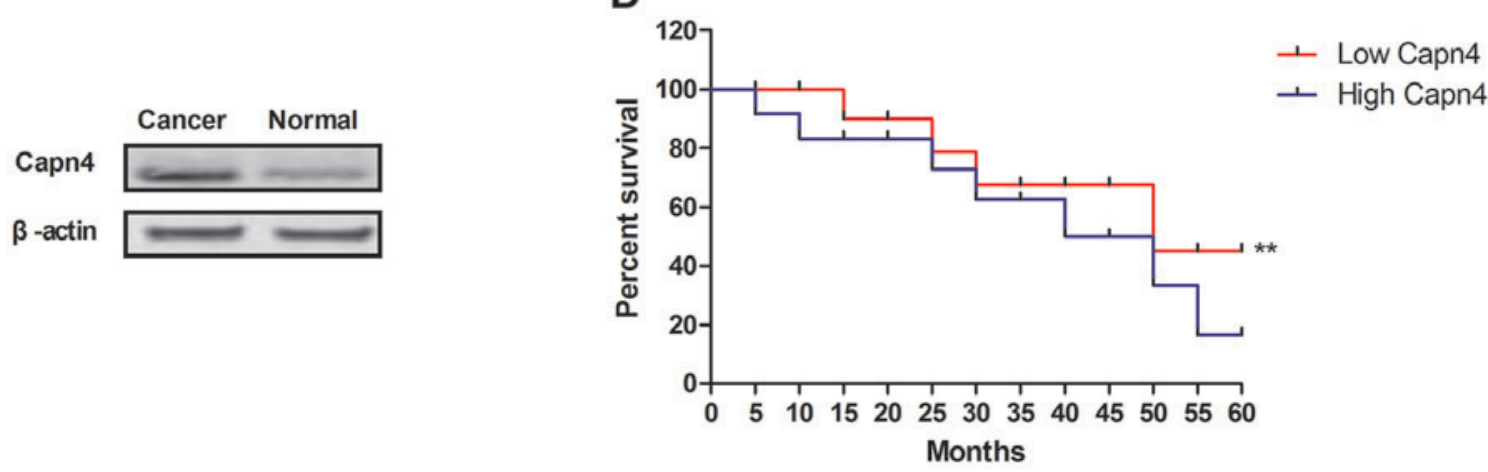

Figure 1. Expression levels and clinical significance of Capn4 in ovarian carcinoma. (A) Capn4 presents increased expression in ovarian carcinoma tissues compared with normal ovarian epithelial tissues. (B) Gene and (C) protein expression levels of Capn4 in ovarian carcinoma tissues and normal ovarian epithelial tissues. (D) Analysis of the association between Capn4 expression and survival of ovarian carcinoma patients. Capn4, calpain small subunit 4 . ${ }^{* *} \mathrm{P}<0.01$ vs. Normal.

at $4^{\circ} \mathrm{C}$. A Ventana Benchmark automated staining system was used for analyzing protein expression (Olympus BX51; Olympus Corporation, Tokyo, Japan). The density of the bands was analyzed using Quantity One software (version 4.62; Bio-Rad Laboratories, Inc., Hercules, CA, USA).

Immunohistochemistry. Paraffin-embedded 4- $\mu \mathrm{m}$ tissue sections were prepared for further analysis. The paraffin sections were treated with $\mathrm{H}_{2} \mathrm{O}_{2}(3 \%)$ for $10-15$ min at $37^{\circ} \mathrm{C}$, which subsequently were blocked by a regular blocking solution (5\% skimmed milk powder in PBST) for 10-15 min at $37^{\circ} \mathrm{C}$. Finally, the sections were incubated with anti-Capn 4 $(1: 1,000)$ at $4^{\circ} \mathrm{C}$ for $12 \mathrm{~h}$. All sections were washed with PBST three times and incubated with HRP-conjugated $\mathrm{IgG}$ (cat. no. PV-6001; OriGene Technologies, Inc.) for $24 \mathrm{~h}$ at $4^{\circ} \mathrm{C}$. The relative expression of Capn 4 was determined in six random views under a light microscope. Images were captured and analyzed using a MicroChemi chemiluminescence system (version 4.2; Eastwin, Shenzen, China).

Statistical analysis. All data are expressed as the mean \pm standard deviation of triplicate dependent experiments and analyzed using Student's t-test or a one-way analysis of variance (followed by Tukey's honest significant difference test). Patients' survival was analyzed using the Kaplan-Meier method. All data were analyzed using SPSS software (version 19.0; IBM Corp., Armonk, NY, USA). P<0.05 was considered to indicate a statistically significant difference.

\section{Results}

Capn4 is overexpressed in ovarian carcinoma and is associated with poor clinical outcomes. To determine the significance of Capn 4 , its expression was analyzed in ovarian carcinoma $(n=5)$ and normal ovarian epithelial tissues from the same patients. The results revealed that Capn 4 was more highly expressed in ovarian carcinoma tissues compared with in normal ovarian epithelial tissues determined by immunohistochemistry (Fig. 1A). The gene and protein expression levels of Capn4 were also increased in ovarian carcinoma tissues compared with normal ovarian epithelial tissues (Fig. 1B and C). It was demonstrated that Capn4 expression was markedly associated with poor survival of patients (Fig. 1D). Taken together, these results indicated that the overexpression of Capn 4 may be associated with the progression of ovarian carcinoma.

Capn4 silencing inhibits the viability and aggressiveness of ovarian carcinoma cells. The function of Capn4 silence was analyzed in ovarian carcinoma cells and scrambled siRNA (siR-vector) was used to exclude the non-specific target effect. The results revealed that Capn 4 is more highly expressed in ovarian carcinoma cells SKOV3 compared with in normal ovarian epithelial cells (IOSE80) (Fig. 2A). It was revealed that Capn4 silencing inhibited ovarian cancer cell (SKOV3) viability in vitro (Fig. 2B). It was also identified that Capn4 silencing markedly inhibited the migration and invasion of SKOV3 cells (Fig. 2C and D). Taken together, these results 
A

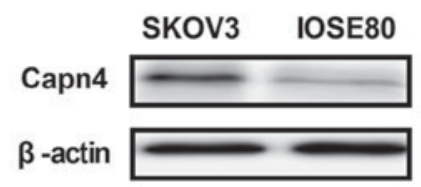

C
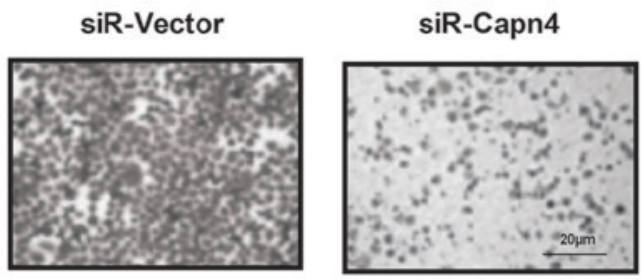

D
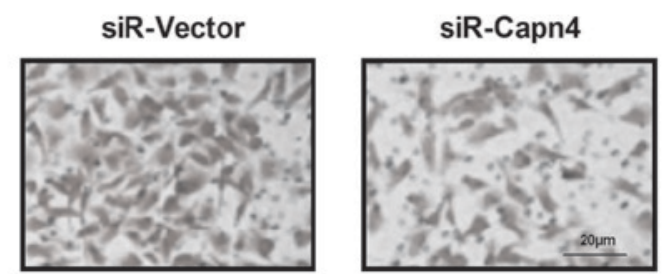

B
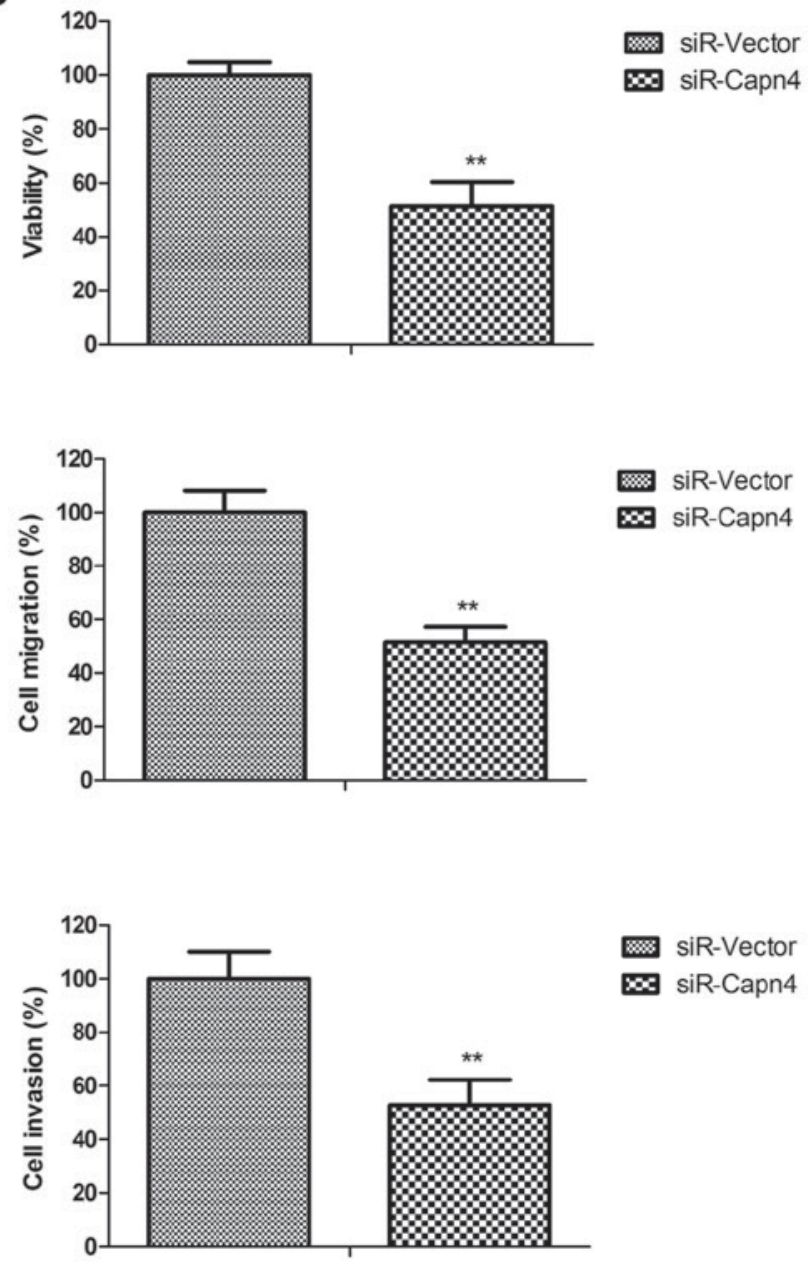

Figure 2. Knockdown of Capn4 inhibits the viability and aggressiveness of ovarian carcinoma cells. (A) Expression levels of Capn4 in ovarian carcinoma cells (SKOV3) and normal ovarian epithelial cells (IOSE80). (B) Capn4 silencing inhibited ovarian cancer cells SKOV3 viability in vitro. Capn4 silencing inhibited (C) migration and (D) invasion of SKOV3 cells. Capn4, calpain small subunit 4; siR, siRNA. ${ }^{* *} \mathrm{P}<0.01$ vs. siR-Vector.

indicated that Capn4 silencing may inhibit the viability and aggressiveness of ovarian carcinoma cells.

Capn4 silencing promotes apoptosis of ovarian carcinoma cells via the caspase-3 apoptotic signaling pathway. To investigate the underlying biological functions of Capn4 in ovarian carcinoma cells, apoptosis was determined in Capn4-silenced ovarian carcinoma cells. Results indicated that Capn 4 silencing promoted the apoptosis of SKOV3 cells induced by cisplatin (Fig. 3A). Capn4 silencing increased the expression levels of the pro-apoptotic caspase- 3 and caspase- 9 compared with the control group (Fig. 3B). It was revealed that Capn4 silencing decreased the expression levels of the anti-apoptotic Bcl-2 and Bcl-w compared with the control group (Fig. 3C). Taken together, these results indicate that Capn 4 silencing may promote apoptosis of ovarian carcinoma cells via the capase-3 apoptotic signaling pathway.

Capn4 silencing inhibits ovarian carcinoma cell metastasis via the OPN-mediated PI3K/AKT signaling pathway. The mechanism mediated by Capn 4 in ovarian carcinoma cells was investigated. The results indicated that Capn4 silencing increased OPN, PI3K and AKT expression in SKOV3 cells
(Fig. 4A). It was identified that Capn4 silencing increased levels of p-PI3K and p-AKT in SKOV3 cells (Fig. 4B). OPN silencing also decreased the expression levels of $\mathrm{p}-\mathrm{PI} 3 \mathrm{~K}$ and p-AKT in SKOV3 cells (Fig. 4C). Results demonstrated that PI3K inhibitor inhibited the migration and invasion of SKOV3 cells in Capn4-silenced SKOV3 cells (Fig. 4D and E). Taken together, these results indicate that Capn4 regulated ovarian carcinoma cell metastasis via the OPN-mediated PI3K/AKT signaling pathway.

\section{Discussion}

Inhibiting systemic metastasis of ovarian cancer may be a novel therapeutic option for patients with ovarian cancer (26). Evidence has indicated that Capn 4 may represent a potential therapeutic target in the treatment of human cancer $(16,27)$. In the present study, Capn4 expression levels were investigated in ovarian cancer tissues and ovarian cancer cell lines in vitro. Previous studies indicated that activation of the $\mathrm{PI} 3 \mathrm{~K} / \mathrm{AKT}$ signaling pathway is involved in the progression of ovarian serous cystadenocarcinoma $(28,29)$. Although it has been demonstrated that Capn 4 enhances OPN expression through activation of the Wnt/ $\beta$-catenin signaling 

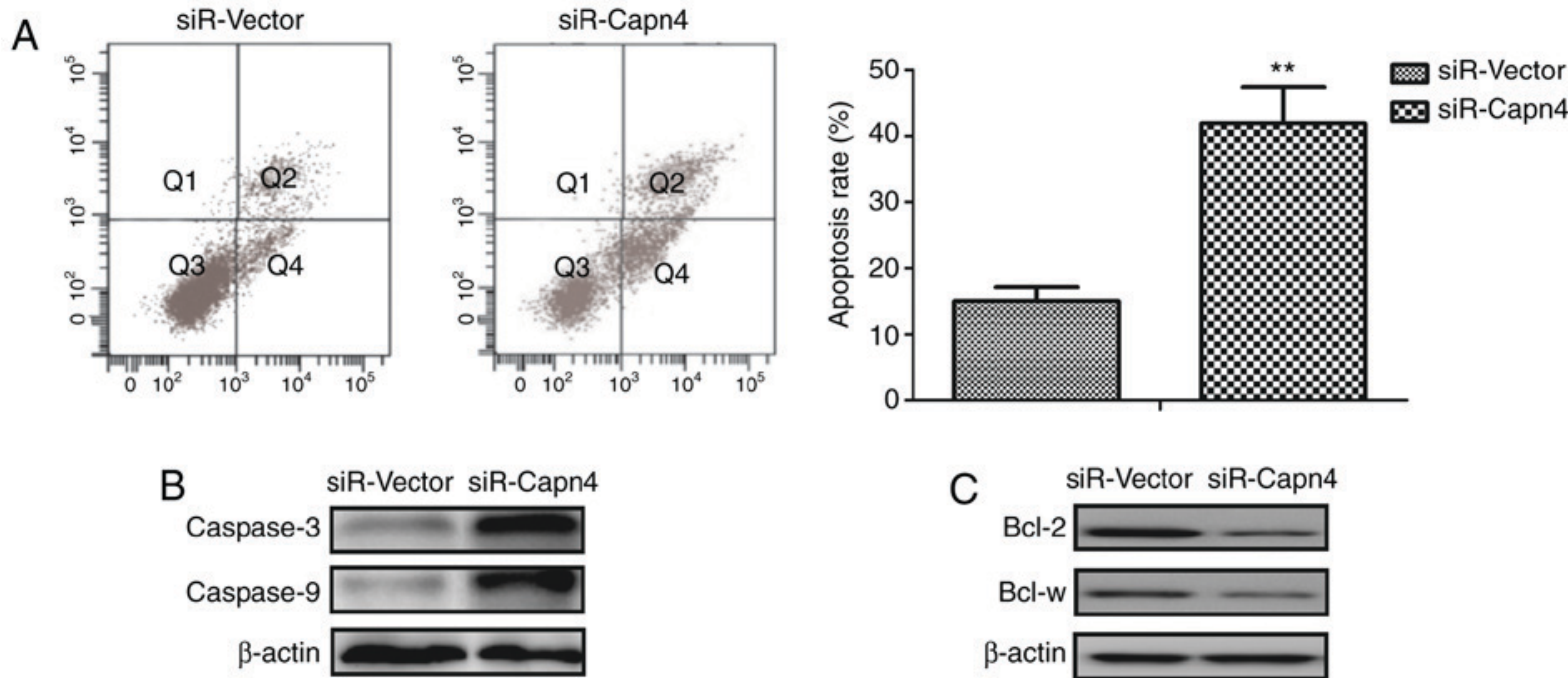

Figure 3. Knockdown of Capn4 promotes apoptosis of ovarian carcinoma cells via the capase-3 apoptosis signaling pathway. (A) Capn4 silencing promoted the apoptosis of SKOV3 cells induced by cisplatin. (B) Capn 4 silencing increased the expression levels of the pro-apoptotic caspase-3 and caspase-9 in SKOV3 cells. (C) Capn4 silencing decreased the expression levels of the anti-apoptotic Bcl-2 and Bcl-w in SKOV3 cells. Capn4, calpain small subunit 4; siR, siRNA; Bcl, B-cell lymphoma; Q, quadrant. ${ }^{* *} \mathrm{P}<0.01$ vs. siR-Vector.

A

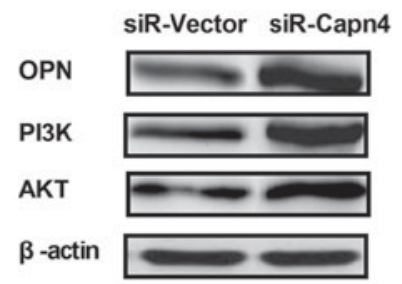

B

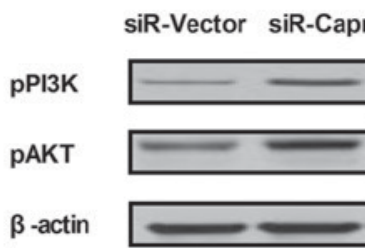

C

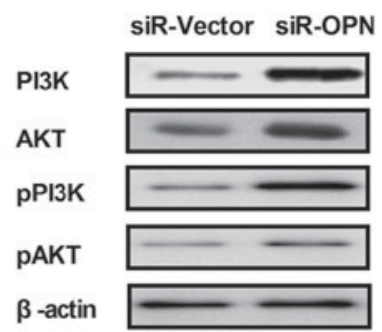

D
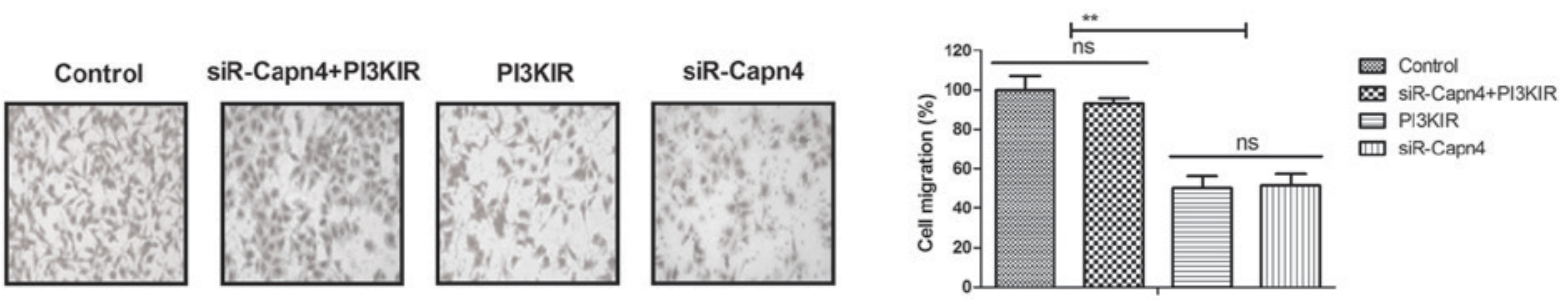

E
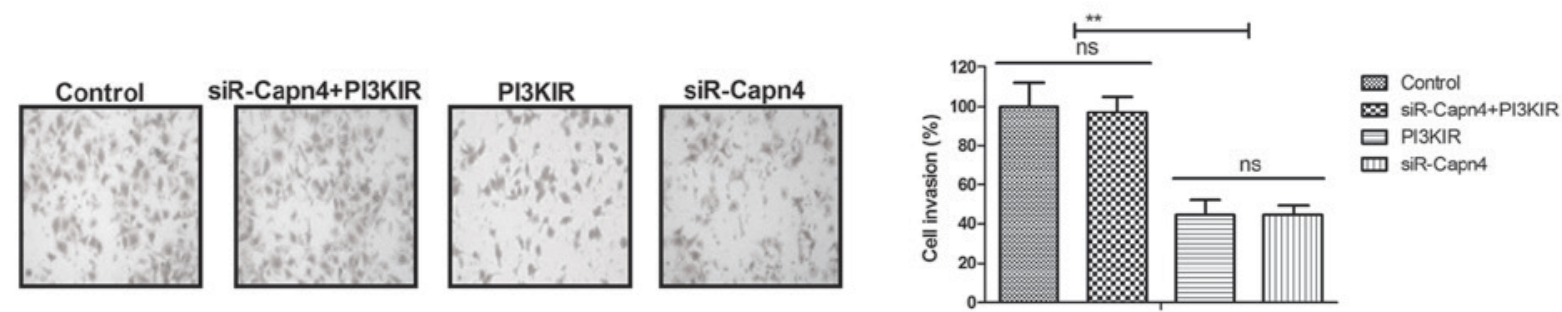

Figure 4. Knockdown of Capn4 inhibits ovarian carcinoma cells metastasis via the OPN-mediated PI3K/AKT signaling pathway. (A) Capn4 silencing increased OPN, PI3K and AKT expression in SKOV3 cells. (B) Capn4 silencing increased the levels of p-PI3K and p-AKT in SKOV3 cells. (C) OPN silencing downregulated the expression and levels of p-PI3K and p-AKT in SKOV3 cells. PI3K inhibitor inhibited (D) migration and (E) invasion of SKOV3 cells in Capn4-silenced SKOV3 cells. Capn4, calpain small subunit 4; siR, siRNA; OPN, osteopontin; PI3K, phosphoinositide 3-kinase; AKT, protein kinase B; p-, phospho-; NS, not significant; PI3KIR, PI3K inhibitor. ${ }^{* *} \mathrm{P}<0.01$ vs. control or siR-Capn4+PI3KIR.

pathway (18), to the best of our knowledge, the association between Capn4 and the PI3K/AKT signaling pathway has not been investigated in ovarian cancer cells. The results of the present study indicated that Capn4 regulated ovarian cancer cell migration and invasion via the PI3K/AKT signaling pathway. 
Targeted therapy has much potential as a treatment approach, as it exhibits lower toxicity to normal cells, but not to tumor cells (30-32). Capn4 is a secreted protein with relevant interactions with numerous migration- and invasion-associated proteins (33). The results of the present study identified that Capn4 is overexpressed in ovarian carcinoma tissues and ovarian carcinoma cells compared with normal ovarian tissues and cells. Capn 4 overexpression increased tumor invasion and metastasis following tumorectomy for hepatocellular carcinoma, which may be a candidate biomarker for future diagnosis and a target for therapy (17). It was revealed that Capn4 exhibited a significant association with poor survival of patients with ovarian carcinoma, and thus may be a potential target for ovarian carcinoma therapy. Notably, Capn4 knockdown significantly inhibited viability and aggressiveness of ovarian carcinoma cells via the OPN-mediated PI3K/AKT signaling pathway.

OPN serves a significant function in a number of physiological and pathological processes, including wound healing, inflammation, immune response and tumor progression (34). OPN overexpression is associated with ovarian cancer progression and tumorigenesis via regulation of the PI3K/AKT signaling pathway (21). In the present study, it was observed that Capn4 knockdown downregulated OPN expression in ovarian cancer cells via downregulation of the PI3K/AKT signaling pathway. The results indicated that the PI3K inhibitor blocked Capn4-OPN-regulated viability and migration of ovarian cancer cells. These results suggest that Capn 4 may be a potential target for the treatment of ovarian cancer. A previous study identified that mitogen-activated protein kinase/extracellular-signal-regulated kinase (ERK) kinase kinase 2/ERK1/2/Capn4 signaling is involved in the migration of breast cancer cells (27). The results of the present study also indicated that the Capn4/OPN/PI3K/AKT signaling pathway participates in the migration and invasion of ovarian carcinoma cells. Additionally, a previous study indicated that Capn4 may represent a potential therapeutic target and a novel prognostic marker of non-small cell lung cancer (16). In the present study, it was identified that Capn 4 is a potential target for the inhibition of ovarian carcinoma cells.

Apoptosis serves an important function in the treatment of cancer (35-37). In the present study, the potential role of Capn4 in the apoptotic signaling pathway was analyzed. A previous study has demonstrated that the overexpression of the apoptosis-associated gene caspase- 3 contributed to the apoptosis of human ovarian cancer cell lines induced by cisplatin (38). The results of the present study revealed that Capn4 silencing promoted the apoptosis of ovarian cancer cells induced by cisplatin by downregulation of Bcl-2 and $\mathrm{Bcl}-\mathrm{w}$ expression. It was indicated that Capn 4 silencing may promote apoptosis of ovarian carcinoma cells via the capase-3 apoptosis signaling pathway. However, a limitation of the present study is that only a single cell line was used to identify the effects of Capn4, as is the small number of ovarian carcinoma samples from patients.

In conclusion, the results of the present study indicated that Capn4 may be a potential target for ovarian carcinoma therapy. The results highlight the regulatory role of Capn4 in the growth and metastasis of ovarian cancer, and reveal that Capn4 knockdown may upregulate the PI3K/AKT signaling pathway via upregulation of OPN expression in ovarian carcinoma cells. Capn4 may be regarded as a candidate therapeutic target for the future treatment of ovarian carcinoma.

\section{Acknowledgements}

Not applicable.

\section{Funding}

No funding received.

\section{Availability of data and materials}

The analyzed data sets generated during the study are available from the corresponding author on reasonable request.

\section{Authors' contributions}

FX designed the experiments. YC performed the experiments. GW, YW, XG, KW, and JL prepared the investigations and analyzed data. All authors have read and approved the final manuscript.

\section{Ethics approval and consent to participate}

The present study was approved by the Ethics Committee of Tianjin Medical University General Hospital (Tianjin, China). Patient provided written informed consent.

\section{Patient consent for publication}

All patients provided written informed consent and approved publication.

\section{Competing interests}

The authors declare that they have no competing interests.

\section{References}

1. Korkmaz T, Seber S and Basaran G: Review of the current role of targeted therapies as maintenance therapies in first and second line treatment of epithelial ovarian cancer; In the light of completed trials. Crit Rev Oncol Hematol 98: 180-188, 2016.

2. Woopen H, Pietzner K, Darb-Esfahani S, Oskay-Oezcelik G and Sehouli J: Extraperitoneal response to intraperitoneal immunotherapy with catumaxomab in a patient with cutaneous lymphangiosis carcinomatosa from ovarian cancer: A case report and review of the literature. Med Oncol 29: 3416-3420, 2012.

3. Dybro A and Blaakær J: Treatment of epithelian ovarian cancer should be followed by lymphadenectomy-a systematic review. Ugeskr Laeger 174: 865-870, 2012 (In Danish).

4. Watts S, Prescott P, Mason J, McLeod N and Lewith G: Depression and anxiety in ovarian cancer: A systematic review and meta-analysis of prevalence rates. BMJ Open 5: e007618, 2015.

5. Davis LL and Carpenter JS: A systematic review of nonpharmacologic interventions for treatment-related symptoms in women with ovarian cancer. Clin J Oncol Nurs 19: 535-542, 2015.

6. McGee J, Panabaker K, Leonard S, Ainsworth P, Elit L and Shariff SZ: Genetics consultation rates following a diagnosis of high-grade serous ovarian carcinoma in the Canadian province of Ontario. Int J Gynecol Cancer 27: 437-443, 2017.

7. Maru Y, Tanaka N, Ohira M, Itami M, Hippo Y and Nagase H: Identification of novel mutations in Japanese ovarian clear cell carcinoma patients using optimized targeted NGS for clinical diagnosis. Gynecol Oncol 144: 377-383, 2017. 
8. Kwon DY, Han GH, Ulak R, Ki KD, Lee JM and Lee SK: Syndrome of inappropriate antidiuretic hormone secretion following irinotecan-cisplatin administration as a treatment for recurrent ovarian clear cell carcinoma. Obstet Gynecol Sci 60: 115-117, 2017.

9. Sirota R, Gibson D and Kohen R: The timing of caffeic acid treatment with cisplatin determines sensitization or resistance of ovarian carcinoma cell lines. Redox Biol 11: 170-175, 2017.

10. Gullbo J, Fryknäs M, Rickardson L, Darcy P, Hägg M, Wickström M, Hassan S, Westman G, Brnjic S, Nygren P, et al: Phenotype-based drug screening in primary ovarian carcinoma cultures identifies intracellular iron depletion as a promising strategy for cancer treatment. Biochem Pharmacol 82: 139-147, 2011.

11. Itamochi H, Kigawa J, Kanamori Y, Oishi T, Bartholomeusz C Nahta R, Esteva FJ, Sneige N, Terakawa N and Ueno NT: Adenovirus type 5 E1A gene therapy for ovarian clear cell carcinoma: A potential treatment strategy. Mol Cancer Ther 6: 227-235, 2007.

12. McKay TR, Bell S, Tenev T, Stoll V, Lopes R, Lemoine NR and McNeish IA: Procaspase 3 expression in ovarian carcinoma cells increases survivin transcription which can be countered with a dominant-negative mutant, survivin T34A; a combination gene therapy strategy. Oncogene 22: 3539-3547, 2003

13. Zhan Q, Wang C and Ngai S: Ovarian cancer stem cells: A new target for cancer therapy. Biomed Res Int 2013: 916819, 2013

14. Ziebarth AJ, Nowsheen S, Steg AD, Shah MM, Katre AA, Dobbin ZC, Han HD, Lopez-Berestein G, Sood AK, Conner M, et al: Endoglin (CD105) contributes to platinum resistance and is a target for tumor-specific therapy in epithelial ovarian cancer. Clin Cancer Res 19: 170-182, 2013.

15. Nakayama K, Nakayama N and Miyazaki K: Development of a novel ovarian cancer molecular target therapy against cancer-related transcriptional factor, NAC1. J Obstet Gynaecol Res 39: 18-25, 2013

16. Gu J, Xu FK, Zhao GY, Lu CL, Lin ZW, Ding JY and Ge D Capn 4 promotes non-small cell lung cancer progression via upregulation of matrix metalloproteinase 2. Med Oncol 32: 51, 2015.

17. Bai DS, Dai Z, Zhou J, Liu YK, Qiu SJ, Tan CJ, Shi YH, Huang C, Wang Z, He YF and Fan J: Capn4 overexpression underlies tumor invasion and metastasis after liver transplantation for hepatocellular carcinoma. Hepatology 49: 460-470, 2009.

18. Yang X, Sun J, Xia D, Can X, Liu L, Zhang J, Xu H, Du N, Liu W, Shen F, et al: Capn4 enhances osteopontin expression through activation of the Wnt//-catenin pathway to promote epithelial ovarian carcinoma metastasis. Cell Physiol Biochem 42: 185-197, 2017.

19. Song Y, Xin X, Xia Z, Zhai X and Shen K: Selective suppression of autocatalytic caspase-3 driven by two-step transcriptiona amplified human telomerase reverse transcriptase promoter on ovarian carcinoma growth in vitro and in mice. Oncol Rep 32: 225-234, 2014

20. Brakora KA, Lee H, Yusuf R, Sullivan L, Harris A, Colella T and Seiden MV: Utility of osteopontin as a biomarker in recurrent epithelial ovarian cancer. Gynecol Oncol 93: 361-365, 2004.

21. Song G, Cai QF, Mao YB, Ming YL, Bao SD and Ouyang GL: Osteopontin promotes ovarian cancer progression and cell survival and increases HIF-1alpha expression through the PI3-K/Akt pathway. Cancer Sci 99: 1901-1907, 2008.

22. Tilli TM, Franco VF, Robbs BK, Wanderley JL, da Silva FR, de Mello KD, Viola JP, Weber GF and Gimba ER: Osteopontin-c splicing isoform contributes to ovarian cancer progression. Mol Cancer Res 9: 280-293, 2011.
23. Hashiguchi Y, Tsuda H, Bandera CA, Nishimura S, Inoue T, Kawamura N, Berkowitz RS and Mok SC: Comparison of osteopontin expression in endometrioid endometrial cancer and ovarian endometrioid cancer. Med Oncol 23: 205-212, 2006.

24. Xiao S, Wang J and Xiao N: MicroRNAs as noninvasive biomarkers in bladder cancer detection: A diagnostic meta-analysis based on qRT-PCR data. Int J Biol Markers 31: e276-e285, 2016.

25. Livak KJ and Schmittgen TD: Analysis of relative gene expression data using real-time quantitative PCR and the 2(-Delta Delta C(T)) method. Methods 25: 402-408, 2001.

26. Eckert MA, Pan S, Hernandez KM, Loth RM, Andrade J, Volchenboum SL, Faber P, Montag A, Lastra R, Peter ME, et al: Genomics of ovarian cancer progression reveals diverse metastatic trajectories including intraepithelial metastasis to the fallopian tube. Cancer Discov 6: 1342-1351, 2016.

27. Li Y, Zhang Z, Zhou X, Li L, Liu Q, Wang Z, Bai X, Zhao Y, Shi H, Zhang $X$ and Ye L: The oncoprotein HBXIP enhances migration of breast cancer cells through increasing filopodia formation involving MEKK2/ERK1/2/Capn4 signaling. Cancer Lett 355: 288-296, 2014

28. Huang Y, Hua K, Zhou X, Jin H, Chen X, Lu X, Yu Y, Zha X and Feng Y: Activation of the PI3K/AKT pathway mediates FSH-stimulated VEGF expression in ovarian serous cystadenocarcinoma. Cell Res 18: 780-791, 2008.

29. Zhou C, Qiu L, Sun Y, Healey S, Wanebo H, Kouttab N, Di W, Yan B and Wan Y: Inhibition of EGFR/PI3K/AKT cell survival pathway promotes TSA's effect on cell death and migration in human ovarian cancer cells. Int J Oncol 29: 269-278, 2006

30. Rodriguez-Brenes IA, Wodarz D and Komarova NL: Quantifying replicative senescence as a tumor suppressor pathway and a target for cancer therapy. Sci Rep 5: 17660, 2015.

31. Collet G, Szade K, Nowak W, Klimkiewicz K, El Hafny-Rahbi B, Szczepanek K, Sugiyama D, Weglarczyk K, Foucault-Collet A, Guichard A, et al: Endothelial precursor cell-based therapy to target the pathologic angiogenesis and compensate tumor hypoxia. Cancer Lett 370: 345-357, 2016.

32. Marech I, Leporini C, Ammendola M, Porcelli M, Gadaleta CD, Russo E, De Sarro G and Ranieri G: Classical and non-classical proangiogenic factors as a target of antiangiogenic therapy in tumor microenvironment. Cancer Lett 380: 216-226, 2016.

33. Dai Z, Zhou SL, Zhou ZJ, Bai DS, Xu XY, Fu XT, Chen Q, Zhao YM, Zhu K, Yu L, et al: Capn4 contributes to tumour growth and metastasis of hepatocellular carcinoma by activation of the FAK-Src signalling pathways. J Pathol 234: 316-328, 2014

34. Hu ZD, Wei TT, Yang M, Ma N, Tang QQ, Qin BD, Fu HT and Zhong RQ: Diagnostic value of osteopontin in ovarian cancer: A meta-analysis and systematic review. PLoS One 10: e0126444, 2015.

35. Mishra J, Drummond J, Quazi SH, Karanki SS, Shaw JJ, Chen B and Kumar N: Prospective of colon cancer treatments and scope for combinatorial approach to enhanced cancer cell apoptosis. Crit Rev Oncol Hematol 86: 232-250, 2013.

36. Braunhut SJ, McIntosh D, Vorotnikova E, Zhou T and Marx KA: Detection of apoptosis and drug resistance of human breast cancer cells to taxane treatments using quartz crystal microbalance biosensor technology. Assay Drug Dev Technol 3: 77-88, 2005.

37. Tilly JL and Kolesnick RN: Sphingolipids, apoptosis, cancer treatments and the ovary: Investigating a crime against female fertility. Biochim Biophys Acta 1585: 135-138, 2002.

38. Yang XK, Xing H, Zheng F, Gao QL, Wang W, Lu YP and Ma D: Role of apoptosis-associated genes and caspase-3 in cisplatin-resistant human ovarian cancer cell lines. Zhonghua Fu Chan Ke Za Zhi 38: 158-161, 2003 (In Chinese). 